\title{
Dynamic properties of metal matrix composites: a comparative study
}

\author{
M. Guden ${ }^{\mathrm{a}}$, I.W. Hall ${ }^{\mathrm{b}, *}$ \\ ${ }^{a}$ Materials Science Program, University of Delaware, Newark, DE 19716, USA \\ ${ }^{\mathrm{b}}$ Department of Mechanical Engineering, University of Delaware, Newark, DE 19716, USA
}

Received 17 March 1997; received in revised form 19 June 1997

\begin{abstract}
Three distinctly different metal matrix composites have been tested at strain rates from quasi-static to $\approx 3000 \mathrm{~s}^{-1}$. It was found that the high strain rate response of each composite was determined primarily by (a) the response of the matrix in the absence of any reinforcement and (b) the damage formation and accumulation processes during deformation. High strain rate behavior of the short fiber composite was dominated by the matrix behavior at low strains but by fiber damage at high strains. The behavior of a whisker reinforced composite was dominated by the matrix properties at all strains. Re-loading tests produced increased fracture strains, indicating that adiabatic heating accelerates fracture of composites by permitting the development of local strain instabilities. () 1998 Elsevier Science S.A.
\end{abstract}

Keywords: Metal matrix composites; Strain rate; Re-loading tests

\section{Introduction}

Compared with their unreinforced alloy counterparts, metal matrix composites (MMCs) usually provide higher strength and modulus, enhanced high temperature strength and wear resistance. Despite the higher manufacturing costs, their outstanding thermo-mechanical properties make MMCs suitable materials in aerospace, defense and automobile industries where improved material performance may outweigh the cost penalty.

MMCs are not a new group of materials and systematic studies of their first-tier thermo-mechanical properties have been ongoing for about 30 years. These studies have provided important design criteria for engineers to use them in structural applications with the necessary high level of confidence. Dynamic loading response is an important design parameter, which is critical in severe applications where impact loading occurs, but has not been greatly investigated to date for MMCs. Under impact conditions, the strain rate in the composite may locally reach strain rates in excess of

* Corresponding author. Tel.: + 1302 8312421; fax: + 1302 8313619.
$1000 \mathrm{~s}^{-1}$, rates which can not be reached using conventional testing methods. The Split Hopkinson Pressure Bar (SHPB), also called the Kolsky Bar, was originally designed by Kolsky [1] to test materials at these moderately high strain rates. The SHPB method is now well established and has been widely used to test conventional metallic materials for about 50 years, and more recently, high strength composite and ceramic materials have also been tested.

Those MMCs which have been tested at high strain rates so far have been diverse in terms of matrix alloy and reinforcement type, size and shape, making comparisons difficult. Harding and Taya [2] carried out tensile tests on $\mathrm{SiC}$ whisker $\left(\mathrm{SiC}_{\mathrm{w}}\right)$ reinforced 2124-T6 aluminum alloy with 15 and 25 volume percentage of fiber $\left(V_{\mathrm{f}}^{\mathrm{f}} \%\right)$ content over a strain rate range of $10^{-3}$ $1500 \mathrm{~s}^{-1}$ and observed that yield stress and fracture strain of the composite increased at high strain rates compared with the quasi-static values. Cho and coworkers [3] measured tensile fracture toughness of a $\mathrm{SiC}_{\mathrm{w}} / 2124-\mathrm{T} 6$ composite containing $13.2 V_{\mathrm{f}}^{\mathrm{o}} \%$ fibers using a modified SHPB set-up and observed a higher fracture toughness under dynamic rather than static loading. Vaziri and co-workers [4] conducted high strain rate compression tests on $\mathrm{SiC}$ particulate $\left(\mathrm{SiC}_{\mathrm{p}}\right)$ 
reinforced 6061-T6 Al alloys over a strain rate range from quasi-static to $\approx 5000 \mathrm{~s}^{-1}$ and found that composites experienced a higher rate sensitivity of the flow stress than their unreinforced alloys. Perng and coworkers [5] conducted high strain rate tensile testing on $\mathrm{Al}_{2} \mathrm{O}_{3}$ particulate reinforced 6061-T6 $\mathrm{Al}$ alloy at different temperatures over a strain rate range of $10^{-3}-\approx$ $400 \mathrm{~s}^{-1}$ and found the ultimate tensile strength and fracture strain of the composite to be more rate sensitive than in the unreinforced alloy. They also observed that the increase of ultimate tensile strength and fracture strain of the composite was more pronounced at increasing temperatures. The same composite material was also tested by Chichili and Ramesh [6] who observed that the tensile fracture strain of the composite increased at high strain rates, $800 \mathrm{~s}^{-1}$, compared with the quasi-static values. Yadav et al. [7] also carried out compression tests on $\mathrm{Al}_{2} \mathrm{O}_{3}$ particulate reinforced 6061T6 Al over a strain rate range of $10^{-4}-10^{5} \mathrm{~s}^{-1}$ using the SHPB and pressure-shear plate impact and observed a greater rate sensitivity of the flow stress of the composite compared with the unreinforced alloy.

The brief summary given above shows that MMCs are more prone to dynamic loading effects than the corresponding unreinforced alloys and the major effects of increasing strain rate on the composites are increasing fracture strain and flow stress. In a recent study Bao and Lin [8] applied finite element analysis to a particulate reinforced alloy and suggested that the main reason for the increasing rate sensitivity of the composite over the matrix alloy is due to the constraining effect of the particles. The constraint increases the local strain rate near the particulate/matrix interface, and hence pushes the composite into the high strain rate regime where the stress in the matrix increases rapidly. However, other reasons for the increased rate sensitivity of the MMCs over their unreinforced alloys have also been given. For example, Yadav et al. [7] proposed two main reasons: (a) change of dislocation density due to dislocation generation and (b) increase of the resistance presented by the particles to the motion of dislocations at dynamic strain rates. A similar reason was given by Perng et al. [5] who proposed that the reduction of the dislocation velocity due to presence of particles necessitated an increase in rate sensitivity of the MMC.

In the present study, compression tests have been conducted on three MMCs using conventional compression SHPB in order to explore their high strain rate mechanical responses and to compare the behavior of three major classes of MMCs, namely, discontinuous fiber-reinforced, whisker-reinforced and particulate-reinforced composites. Analyses of rate sensitivity of the composites are made in comparison with unreinforced alloy properties. The ultimate goal of this type of study is the development of constitutive equations to permit the prediction of the rate sensitive mechanical properties of the MMCs relative to those of the matrix.

\section{Testing methods}

High strain rate tests were conducted using a compression SHPB, comprising three $19 \mathrm{~mm}$ diameter Inconel-718 bars: a striker bar (724 $\mathrm{mm}$ in length), an incident bar (3660 $\mathrm{mm}$ in length) and a transmitter bar (1830 mm in length). The characteristic time window is $\approx 290 \mu$ s. Details of testing can be found elsewhere [9] but, briefly, the striker bar produces a constant amplitude elastic compressive wave in the incident bar; this wave propagates down the bar to the bar/specimen interface where it is partly reflected back into the incident bar as a tensile pulse and partly transmitted to the transmitter bar as a compressive pulse. The reflected pulse $\left(\varepsilon_{\mathrm{r}}\right)$ yields information on the strain rate involved in the specimen and the transmitted wave $\left(\varepsilon_{t}\right)$ gives the stress in the specimen. The stress $(\sigma)$, strain $(\varepsilon)$ and strain rate $(\dot{\varepsilon})$ in the specimen are given by;

$$
\begin{aligned}
& \sigma_{\mathrm{s}}(t)=\frac{A_{\mathrm{b}} E_{\mathrm{b}} \varepsilon_{\mathrm{t}}}{A_{\mathrm{s}}} \\
& \varepsilon=-\frac{2 C_{\mathrm{b}}}{L_{\mathrm{s}}} \int_{0}^{\tau} \varepsilon_{\mathrm{r}} \mathrm{d} t \\
& \dot{\varepsilon}(t)=-\frac{2 C_{\mathrm{b}}}{L_{\mathrm{s}}} \varepsilon_{\mathrm{r}}
\end{aligned}
$$

$A, L, E$ and $C$ are cross-sectional area, length, modulus and elastic wave velocity respectively, and $\mathrm{s}$ and $\mathrm{b}$ refer to the specimen and bar respectively. For the equations to be used for SHPB testing, the following assumptions must be valid: (a) the material deforms homogeneously during the test; (b) the wave propagates through the bars with minimal dispersion and, finally, (c) the stress in the bars never exceeds the elastic limit of the bar material. More information on these assumptions and their effects on SHPB test results can be found elsewhere $[10,11]$. It must, however, be emphasized here that the initial region $(4-6 \mu \mathrm{s})$ of the stress/strain curves corresponding to the elastic region cannot be accurately described by these equations because finite time is required in order to establish stress equilibrium in the specimen $[12,13]$.

Two further techniques were applied during SHPB testing in this study. First, longitudinal strain during the test was restricted using, loose-fitting, high strength steel collars placed around the cylindrical test pieces. The lengths of the collars were selected to allow the desired amount of strain and their diameter was sufficiently larger than the sample that no radial constraint was produced. These tests provided samples tested to the same strain but at different strain rates for microscopic observations. The second technique was to reload, in the SHPB, samples that had already been pre-strained to a level determined either by the collars or by selection of the striker bar velocity. By monitoring relaxation processes which occurred due to inter- 
ruption of the test, this technique was used to detect the effect of sample heating during single shot tests.

The material was cut into parallel-faced plates which were polished to $3 \mu \mathrm{m}$ before core drilling cylindrical samples of the desired diameter (see Table 1). Quasi-static compression tests were conducted using a screw-driven Instron machine and strain gages were mounted on the sample sides. Strain values were obtained from strain gages until they detached from the sample and larger strains were read from the machine output. Similar specimen sizes were used both in quasi-static and high strain rate tests and $\mathrm{MoS}_{2}$ lubricant was used in all cases. Barreling, which can arise due to frictional forces during compression, was not observed in any specimen tested at either quasi-static or high strain rate.

\section{Materials}

The three different MMCs tested were as follows.

\subsection{Short fiber (SF)-reinforced $M M C$}

The first composite was an $\mathrm{Al}-1.2 \mathrm{wt} . \% \mathrm{Cu}$ alloy reinforced with $20 V_{\mathrm{f}}^{\mathrm{o}} \% \delta$ - $\mathrm{Al}_{2} \mathrm{O}_{3}$ short Saffil ${ }^{\mathrm{TM}}$ fibers, $\approx$ $500 \mu \mathrm{m}$ in length and $3-4 \mu \mathrm{m}$ in diameter. This composite material was produced by Honda R\&D, Japan. Composites were manufactured as discs $20 \mathrm{~mm}$ thick and $100 \mathrm{~mm}$ in diameter by a squeeze casting (pre-form) process using a $\mathrm{SiO}_{2}$ binder to provide rigidity to the pre-form. An advantage of this technique is that the use of pressure during casting results in a relatively pore-free microstructure.

Fig. 1(a) shows the typical fiber distribution of MMCs in the planar random plane (PRP), normal to the pressure direction used in the casting process. Within this plane the fibers are randomly oriented but may be slightly inclined to the normal to the pressing direction. Compression testing was carried out normal to the PRP, referred to as the $\mathrm{N}$ direction, and parallel to the planar random plane, referred to as the $\mathrm{P}$ direction. Unreinforced $\mathrm{Al}-1.2 \% \mathrm{Cu}$ alloy, manufactured by the same route, was also tested.

\subsection{Whisker (W)-reinforced $M M C$}

The second composite was in the form of thin plate $5.5 \mathrm{~mm}$ in thickness and contained $25 \mathrm{~V}_{\mathrm{f}}^{\mathrm{o}} \% \mathrm{SiC}_{\mathrm{w}}$ in a 2124-T6 Al matrix alloy. The whiskers were $2-10 \mu \mathrm{m}$ in length with a diameter of $0.1-0.5 \mu \mathrm{m}$ and the composite was manufactured by a $\mathrm{p} / \mathrm{m}$ route involving hot consolidation, extrusion and cross-rolling. Reinforcement distribution in the MMC was again observed to be almost random in the rolling plane. The microstructure of the MMC in the PRP is shown in Fig. 1(b). Compression testing was performed normal to this plane. Unreinforced 2024-T6 alloy (essentially identical in composition to 2124) manufactured by the same route was also tested for comparison.
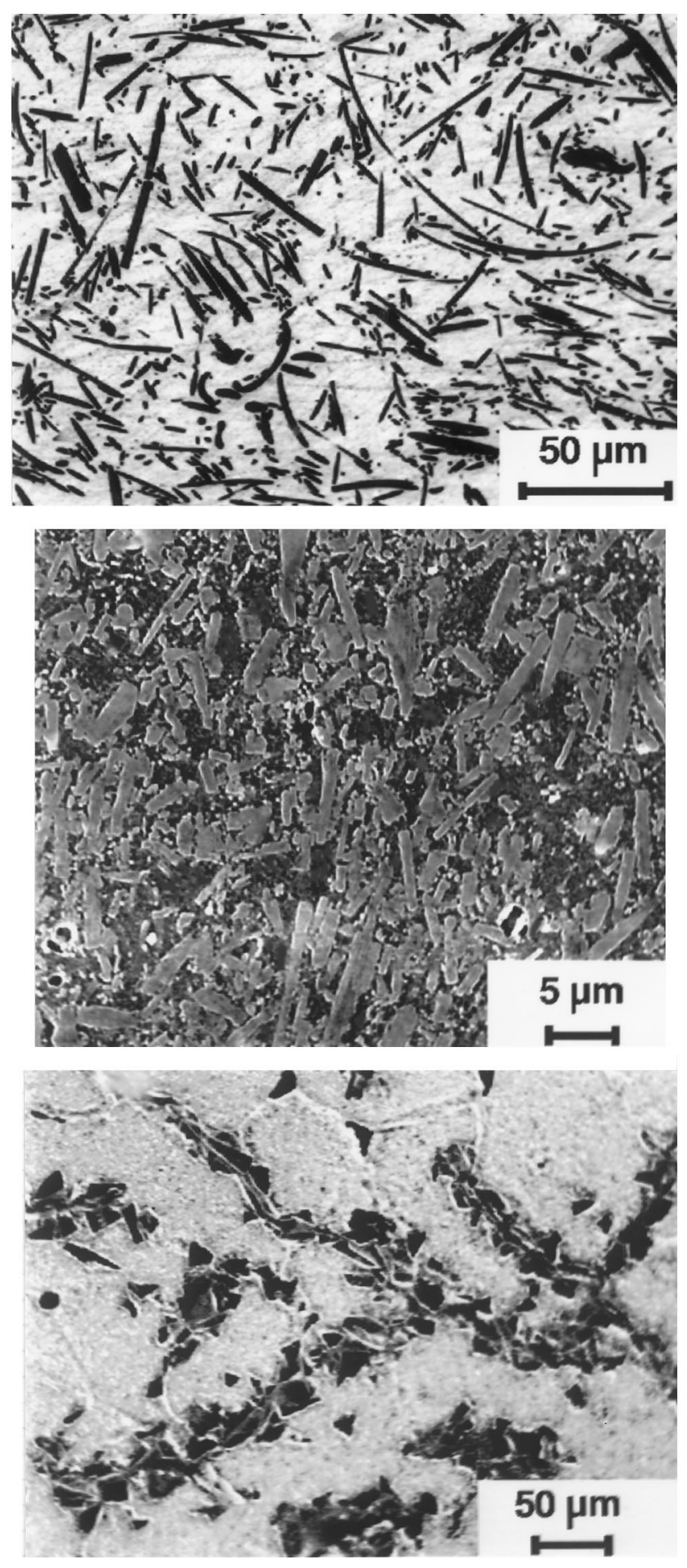

Fig. 1. (a) Fiber distribution in planar random plane of SF composite. (b) Fiber distribution in PRP of $\mathrm{SiC}_{\mathrm{w}} / 2124-\mathrm{T} 6$ composite. (c) Particle distribution in $\mathrm{SiC}_{\mathrm{p}} / 2024-\mathrm{O}$ composite. 


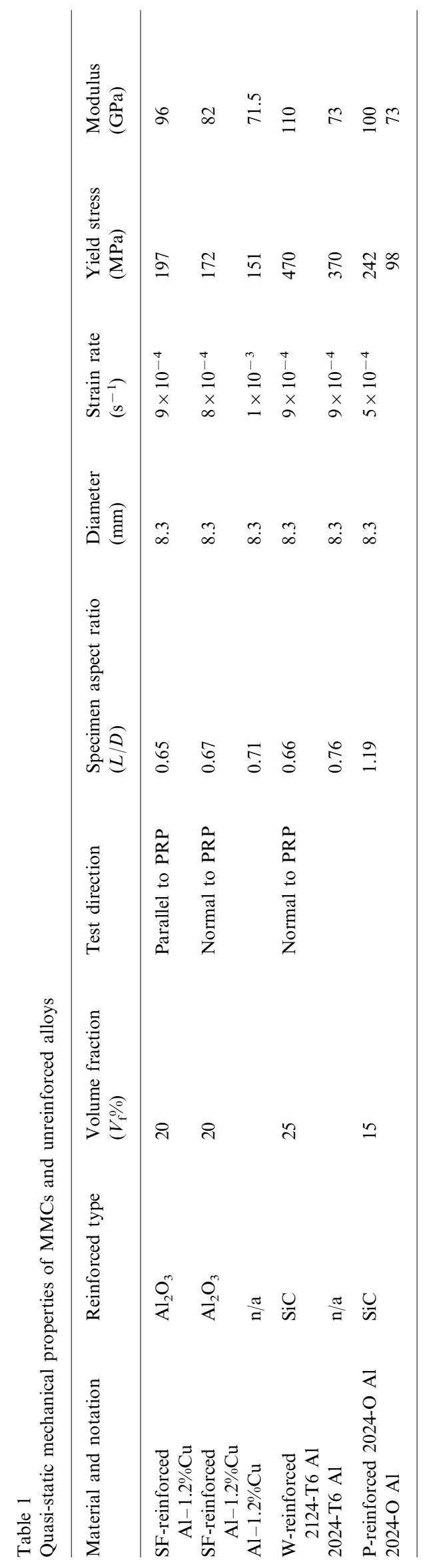




\subsection{Particulate (P)-reinforced $M M C$}

The particulate reinforced composite contained $15 V_{\mathrm{f}}^{\mathrm{o}} \mathrm{SiC}$ particles $20 \mu \mathrm{m}$ in diameter in a matrix of $2024 \mathrm{Al}$. The MMC was prepared by a casting process and the particles were found to be concentrated at cell or dendrite boundaries as shown in Fig. 1(c). Considerable porosity was also present but it will be seen that this did not influence the mechanical properties measured in this study. Data for quasi-static mechanical properties [14] and strain rate sensitivity of the unreinforced 2024 [15] as cast material have been taken from the literature.

\section{Results}

\subsection{Mechanical properties}

Table 1 presents quasi-static mechanical properties of the MMCs and unreinforced alloys from strain gaged samples. The values are the average of at least three separate tests. It should also be noted that, because of material limitations on specimen size, the aspect ratios were not standard: nevertheless, although absolute values may be slightly different, the results do allow for comparison between monolithic alloys and composites tested with similar aspect ratios. The reinforcement effect in the MMCs is evident from the $0.2 \%$ offset yield stress (proof stress) and modulus data.

In the following sections, high strain rate test results are presented separately for each MMC.

\subsubsection{Short fiber-reinforced $M M C$}

Typical stress/strain curves of SF-reinforced composite tested in $\mathrm{P}$ and $\mathrm{N}$ directions at high strain rates, as well as data from the unreinforced alloy, are presented in Fig. 2(a) which shows the dramatic strengthening effect of the fibers, particularly at low strains. Fig. 2(b) shows data for the $P$ direction at increasing strain rates; the flow stress increases with strain and strain rate and a high work hardening rate is maintained until about $5 \%$ strain. Similar but less marked behavior was noted for the $\mathrm{N}$ direction composite. By contrast, the unreinforced matrix showed a steadily increasing flow stress at all strains.

Fig. 2(b) shows that, in the region after $\approx 5 \%$ strain, the work hardening rate in the composite decreased with increasing strain rates. This effect can be more clearly seen in Fig. 3, in which the flow stresses at 5 and $15 \%$ strain are plotted as a function of the logarithm of strain rate for the unreinforced alloy and $\mathrm{P}$ orientation composite, along with $5 \%$ strain data for the $\mathrm{N}$ orientation composite. Linear interpolation yielded a good fit to the stress data shown in Fig. 3. Clearly, at 5\% strain the MMC demonstrated a significantly higher strain
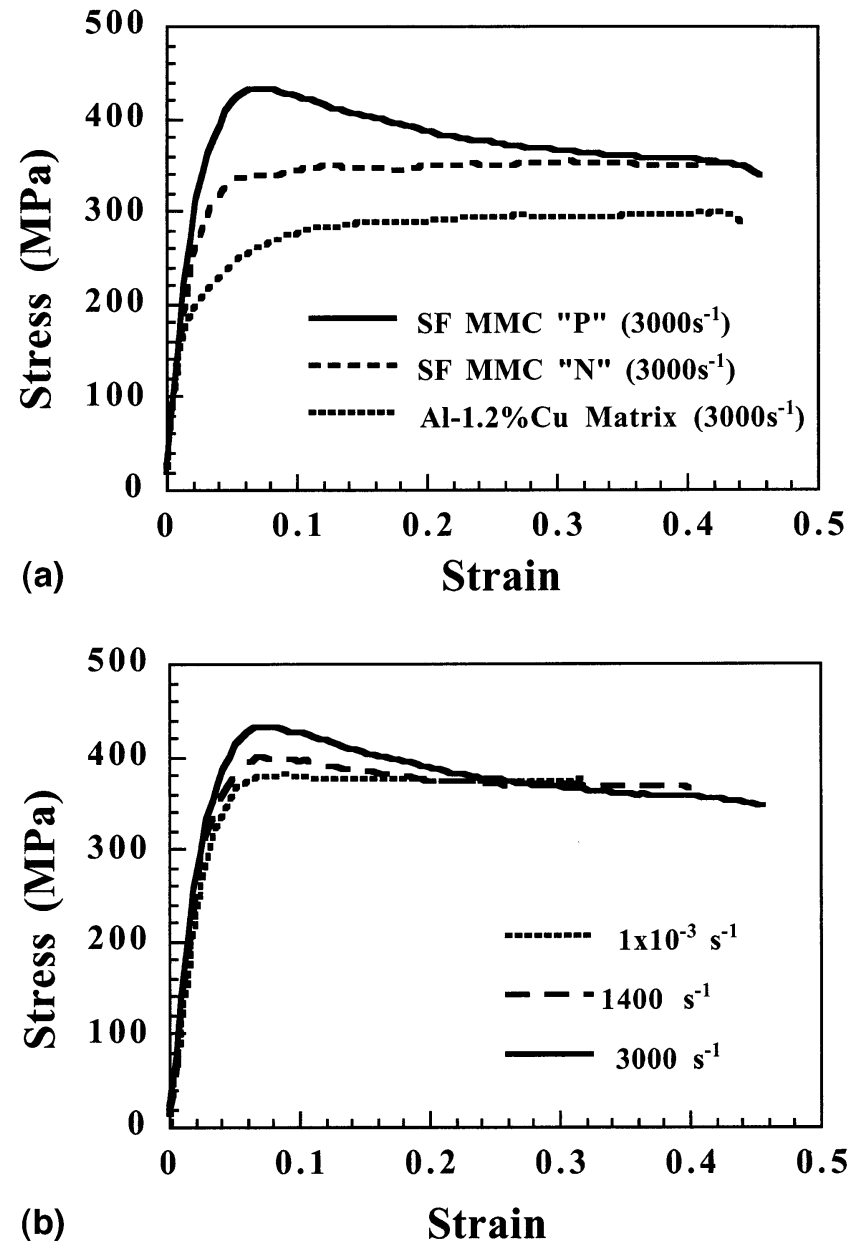

Fig. 2. (a) Stress/strain curves at high strain rate for $\mathrm{P}$ and $\mathrm{N}$ composites and unreinforced $\mathrm{Al}-1.2 \% \mathrm{Cu}$ alloy. (b) Stress/strain curves for $\mathrm{P}$ composite at increasing strain rates.

rate sensitivity, as reflected in the slopes of the linear interpolated curves after $\approx 10^{3} \mathrm{~s}^{-1}$, compared with the unreinforced alloy. Also, MMC tested in the P direction showed higher rate sensitivity as compared with the $\mathrm{N}$ direction. However, as the strain increased from 5 to $15 \%$, the work hardening rate of the MMC decreased compared with the initial high work hardening region and a negative work hardening rate was observed at high strain rates in the $\mathrm{P}$ direction. Reduced work hardening rate with increasing strain rates was also observed in MMC samples tested in the $\mathrm{N}$ direction, but the effect was less pronounced. Note also that, at $15 \%$ strain, the rate sensitivity of the MMC in both directions approached that of the matrix, Fig. 3. Scatter in the stress/strain data values of the MMC is seen to be a little larger than for the unreinforced alloy, most likely due to minor relative differences in the fiber content of the samples tested since small specimens were used.

The re-loading or interrupted tests mentioned above were applied to both MMC and unreinforced alloy in 


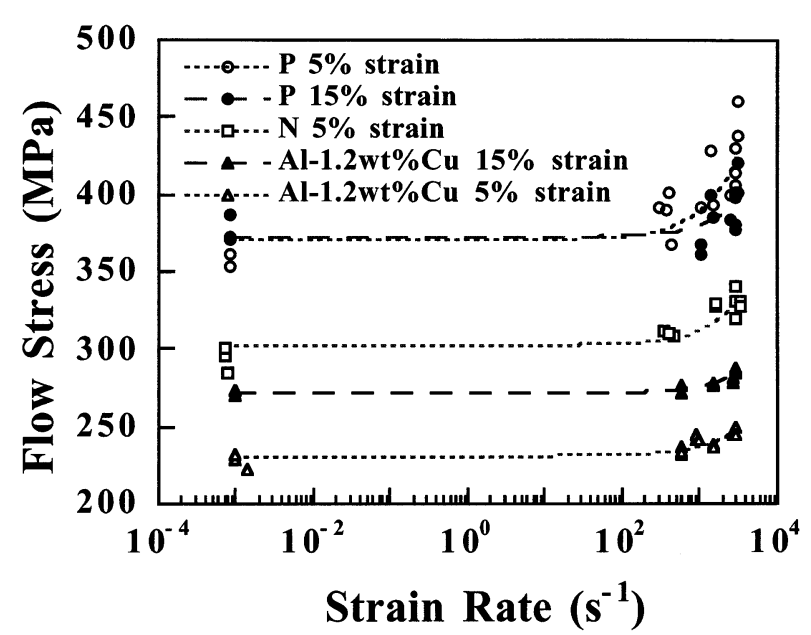

Fig. 3. Flow stresses of $\mathrm{P}$ and $\mathrm{N}$ composites and unreinforced $\mathrm{Al}-1.2 \% \mathrm{Cu}$ matrix at various strains as a function of logarithm of strain rate.

order to see more clearly the effect of increasing strain rate on the stress values of the MMC and unreinforced alloy. Fig. 4 shows the interrupted test behavior of MMC P composite. In these tests, after initial deformation which produced $9-15 \%$ strain, depending on the strain rate, the specimens were allowed to cool to room temperature and then re-loaded at either the same or a higher strain rate. When the initial lower strain rate test was followed by a higher strain rate test a clear initial increase in flow stress values was repeatably observed in the MMC but the flow stress decreased to the low strain rate value after a further $10 \%$ deformation. This shows that considerable sample heating occurred, accompanied by a decrease in flow stress, during testing at elevated strain rates. A similar effect of increased strain rate during re-loading tests was observed for the unreinforced alloy.

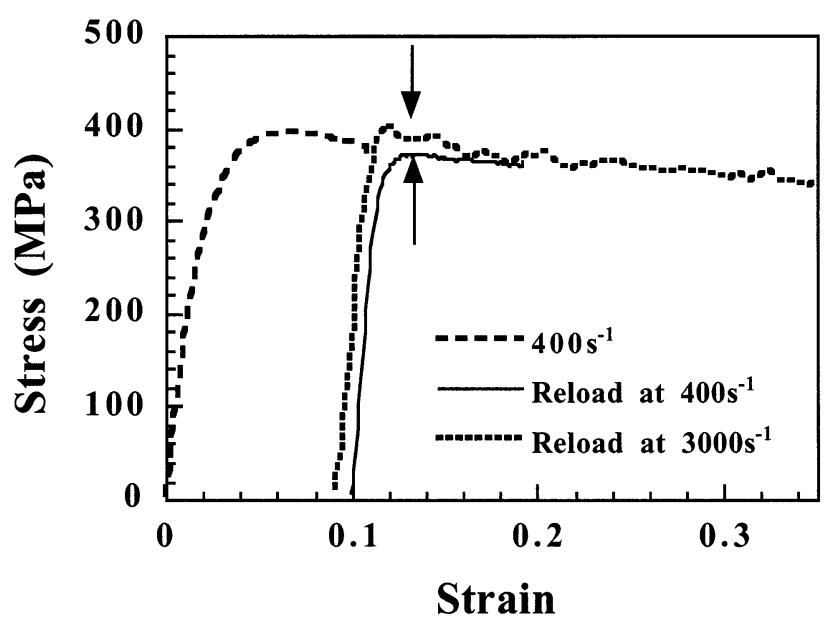

Fig. 4. P composite showing increase in flow stress (arrowed) upon re-loading at higher strain rate.
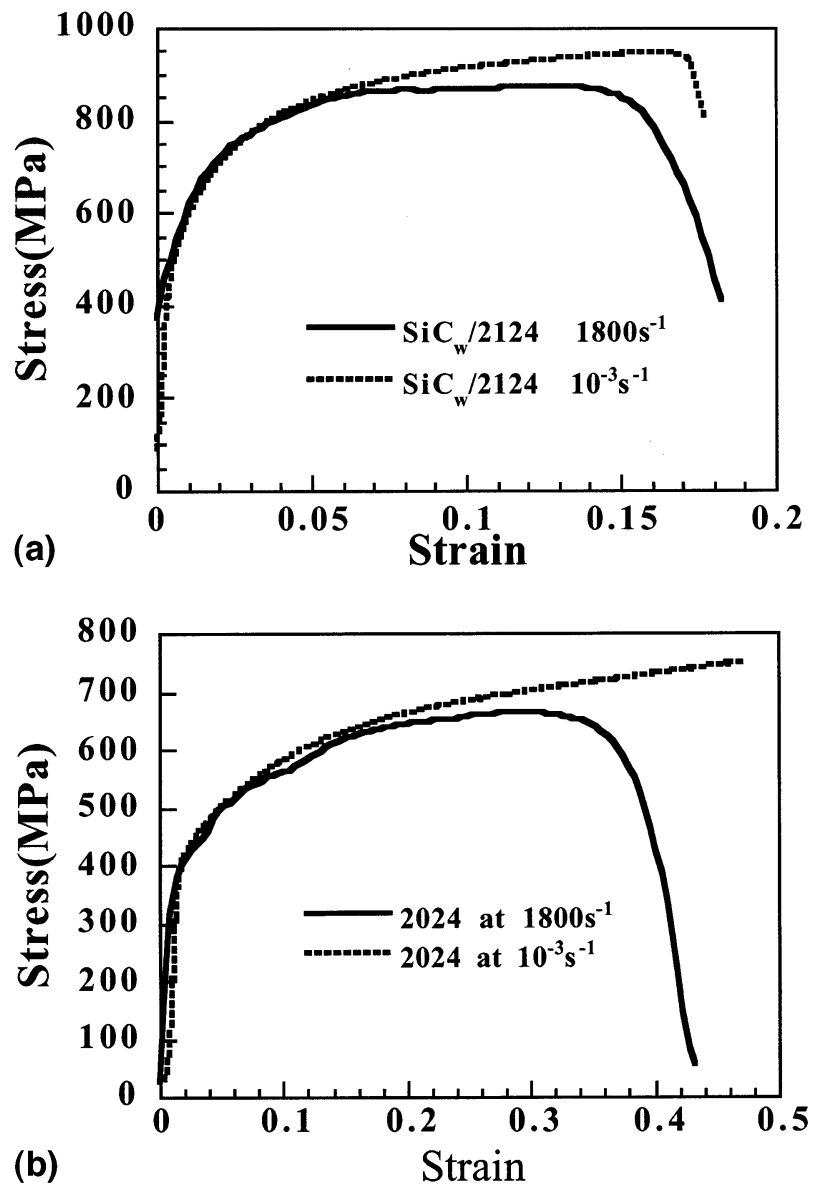

Fig. 5. (a) Stress/strain curves at increasing strain rates for $\mathrm{SiC}_{\mathrm{w}} /$ 2124-T6 composite. (b) Stress/strain curves at increasing strain rate for unreinforced 2024-T6.

\subsubsection{Whisker-reinforced $M M C$}

Typical stress/strain curves of the $\mathrm{SiC}_{\mathrm{w}} / 2124$ and unreinforced alloys at increasing strain rates are shown in Fig. 5(a) and (b) respectively. It was observed that in both materials, $\mathrm{SiC}_{\mathrm{w}} / 2124$ and 2024-T6, the flow stress values at quasi-static and high strain rates were similar at small strain values but that the high strain rate values decreased gradually, relative to quasi-static values, at increasing strain levels. This is believed to be due to the effect of thermal softening at high strain rates since the shorter deformation times allow less time for dissipation of the heat generated. All $\mathrm{SiC}_{\mathrm{w}}$ samples tested at either quasi-static or high strain rates fractured within the strain range of $15-22 \%$ strain. No fracture was observed until about $70 \%$ strain in 2024T6 alloy samples tested at quasi-static strain rates. However, high strain rate tested samples of 2024-T6 alloy fractured at a strain of $\approx 35 \%$. Note that stress maxima, a common indication of shear-band formation, were observed in the true stress/strain curves of both the $\mathrm{SiC}_{\mathrm{w}} \mathrm{MMC}$ and unreinforced alloy tested at high strain rates. 
The MMCs fracture strain decreased very slightly with increasing strain rate, between quasi-static $(18 \%$ on average) and $2800 \mathrm{~s}^{-1}(\approx 15 \%$ on average). A similar effect of strain rate on the fracture strain of the unreinforced 2024-T6 alloy was observed in the high strain rate regime, resulting in fracture strains of $\approx$ $33 \%$ at $3000 \mathrm{~s}^{-1}$.

The flow stress values of the MMC and unreinforced 2024-T6 alloy at 5\% strain were essentially independent of strain rate, Fig. 6. At 15\% strain, the dynamic flow stress values were slightly reduced compared with the respective quasi-static values for both materials. The flow stress values of unreinforced 2024-T6 alloy at 15\% strain were almost constant within the studied high strain rate region, $600-3200 \mathrm{~s}^{-1}$, but they were slightly lower than quasi-static values. Note also, the reduction of flow stress at $15 \%$ strain from quasi-static to dynamic strain rates was higher in the MMC, $\approx 70 \mathrm{MPa}$, than in unreinforced 2024-T6 alloy, $\approx 20 \mathrm{MPa}$.

Interrupted tests at the same strain rate were applied to MMC and 2024-T6 samples and typical results of these studies are shown in Fig. 7(a) and (b) for strain rate of $\approx 1700 \mathrm{~s}^{-1}$. Fig. 7(a) shows the effect of a test interrupted at $\approx 8 \%$ strain in the MMC. It is seen that re-loading did not affect flow stress but did increase the fracture strain. In single shot tests at $\approx 950 \mathrm{~s}^{-1}$ the average fracture strain was $17.9 \%$, while for tests interrupted at $8 \%$ strain it was $23.5 \%$. Likewise at $\approx 1700$ $\mathrm{s}^{-1}$, the average fracture strain was $17.3 \%$ for single shot tests, while interrupted tests increased the average fracture strain to $22.5 \%$. Within the studied strain rate range, increasing strain rate during re-loading produced very similar results in all these MMCs. Interrupted tests conducted on 2024-T6 unreinforced alloy, Fig. 7(b), also showed that re-loading after $\approx 20 \%$ strain produced almost no effect on the flow stress values but did greatly increase the fracture strain. The fracture strains

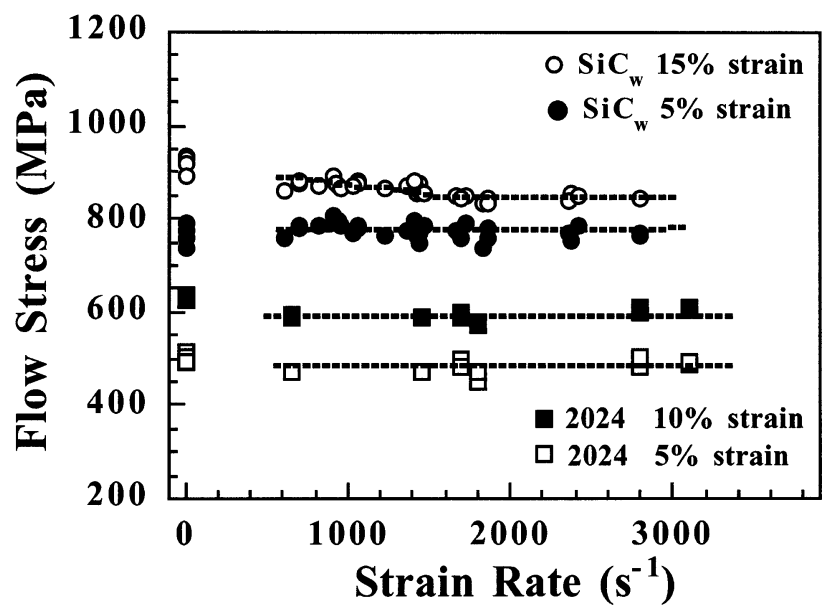

Fig. 6. Flow stresses of $\mathrm{SiC}_{\mathrm{w}} / 2124-\mathrm{T} 6$ and $2023-\mathrm{T} 6$ as a function of strain rate.

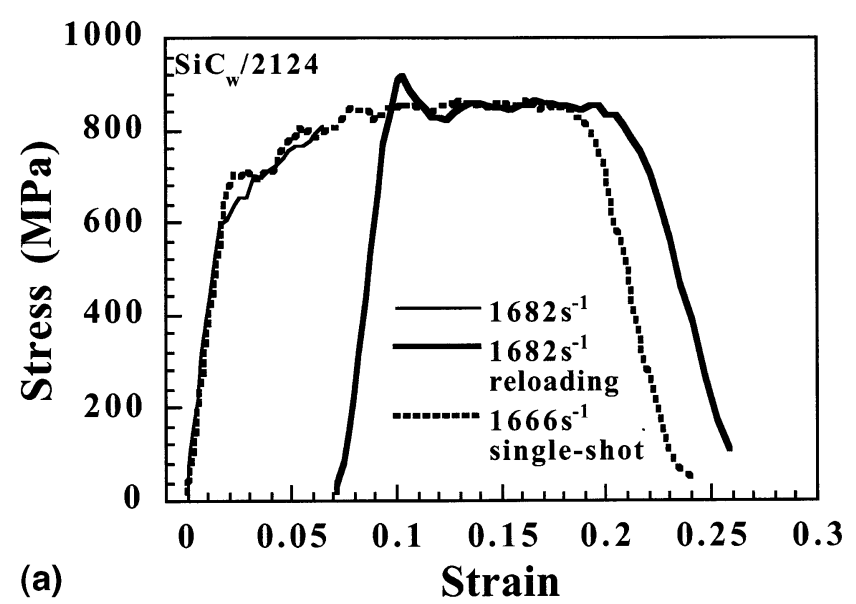

(a)

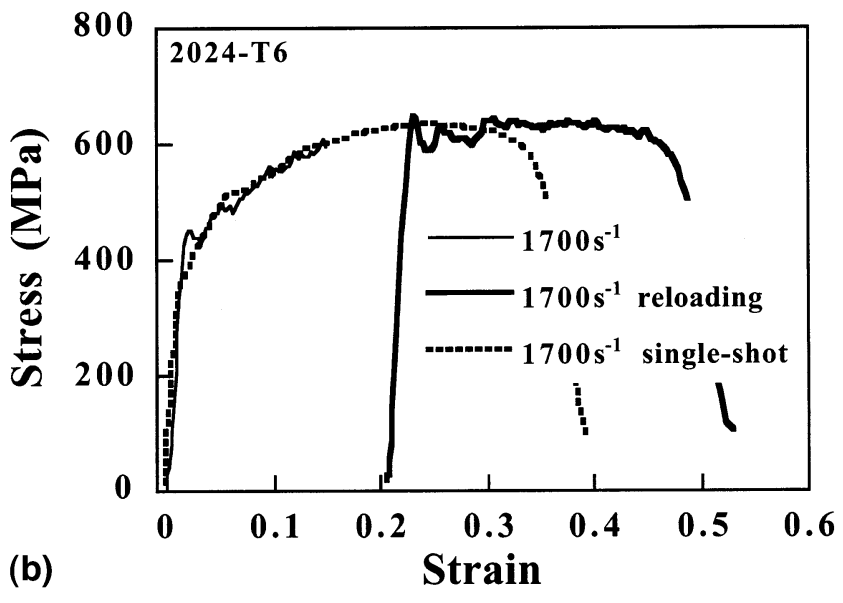

Fig. 7. (a) Stress/strain curves showing re-loading tests for $\mathrm{SiC}_{\mathrm{w}} / 2124-$ T6. (b) Stress/strain curves from re-loading test on 2024-T6 alloy.

increased from 35 to $46 \%$ between single shot and interrupted tests at $\approx 1700 \mathrm{~s}^{-1}$. These results essentially confirm that the $\mathrm{SiC}_{\mathrm{w}} / 2124-\mathrm{T} 6$ and 2024-T6 unreinforced alloy show similar trends in terms of the mechanical properties studied here.

\subsubsection{Particle-reinforced $M M C$}

Typical stress/strain curves for the MMC are shown in Fig. 8(a) for different strain rates showing that, in this case, the flow stresses increased with increasing strain rate as well as with increasing strain. The MMC was found to be rate sensitive within the studied strain rate and strain regimes. Fig. 8(b) presents the flow stress as a function of the logarithm of strain rate and confirms that, in contrast to the $\mathrm{SiC}_{\mathrm{w}} / 2124$ composite, the strain rate sensitivity is retained even at high strains. These specimens did not fracture even at the highest strain rates and at strains in excess of $20 \%$.

\subsection{Microscopy}

It is known that damage accumulation can lead to well defined unloading (relaxation) processes in MMCs 
and the extent of these processes varies with several factors including fiber size and strength, matrix strength and properties of fiber/matrix interface. Since the MMCs studied are quite different in terms of these parameters, the expected damage processes will also be quite different in each. The following sub-sections report the results of microscopical characterization of these damage processes for each MMC separately and these are subsequently related to the measured mechanical property response.

\subsubsection{Short fiber-reinforced $M M C$}

No fracture was observed in unreinforced Al$1.2 \% \mathrm{Cu}$ alloy until about $70 \%$ strain. Although complete fracture of the specimen was not observed in the MMC samples, cracks were seen along the surface of the cylindrical specimens tested in the $\mathrm{P}$ direction to large strains, Fig. 9(a).

Longitudinal sections of samples tested at quasistatic and dynamic strain rates to small $(\approx 2 \%)$ and large $(\approx 36 \%)$ strains were prepared for optical and scanning electron microscopy (SEM). Observations
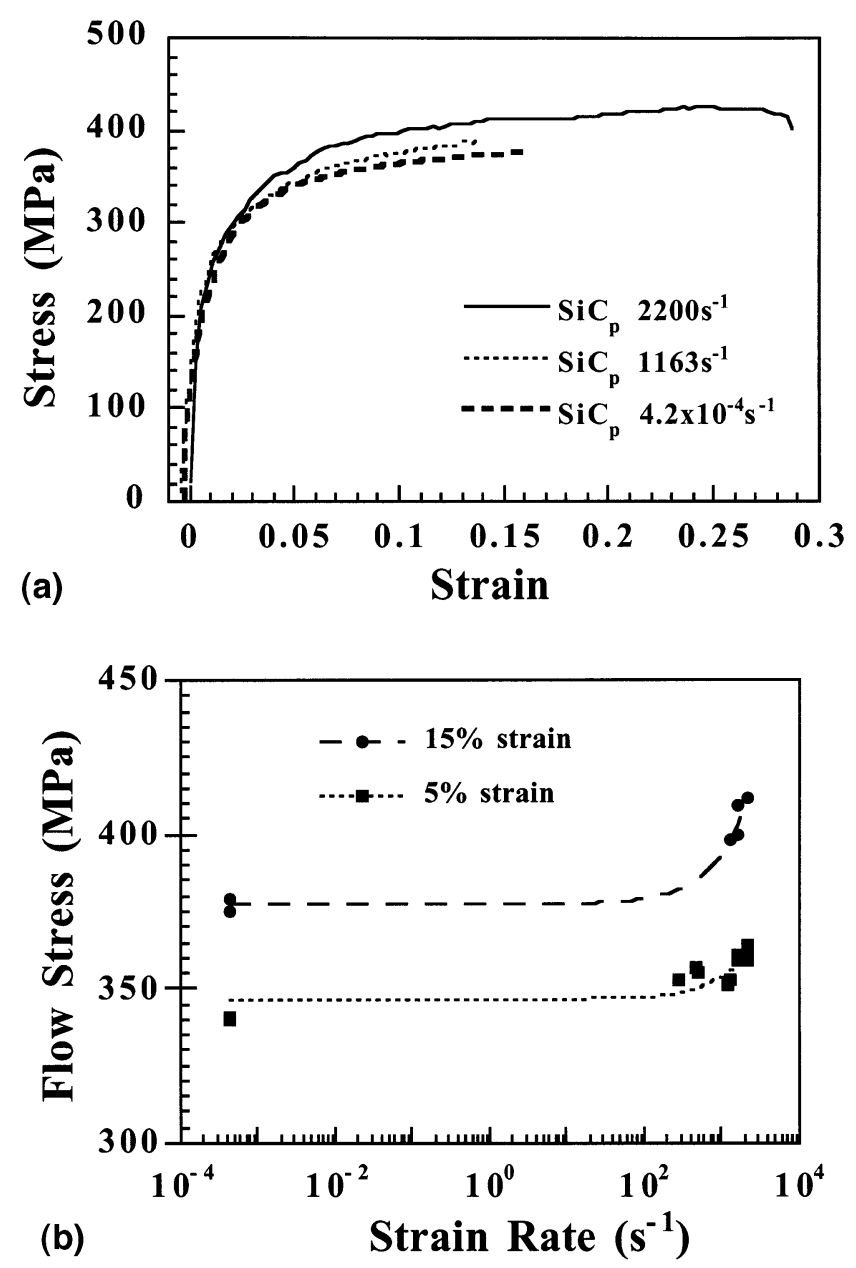

Fig. 8. (a) Stress/strain curves for $\mathrm{SiC}_{\mathrm{p}} / 2024-\mathrm{O}$ composite. (b) Flow stress vs. logarithm of strain rate for $\mathrm{SiC}_{\mathrm{p}} / 2024-\mathrm{O}$ composite.
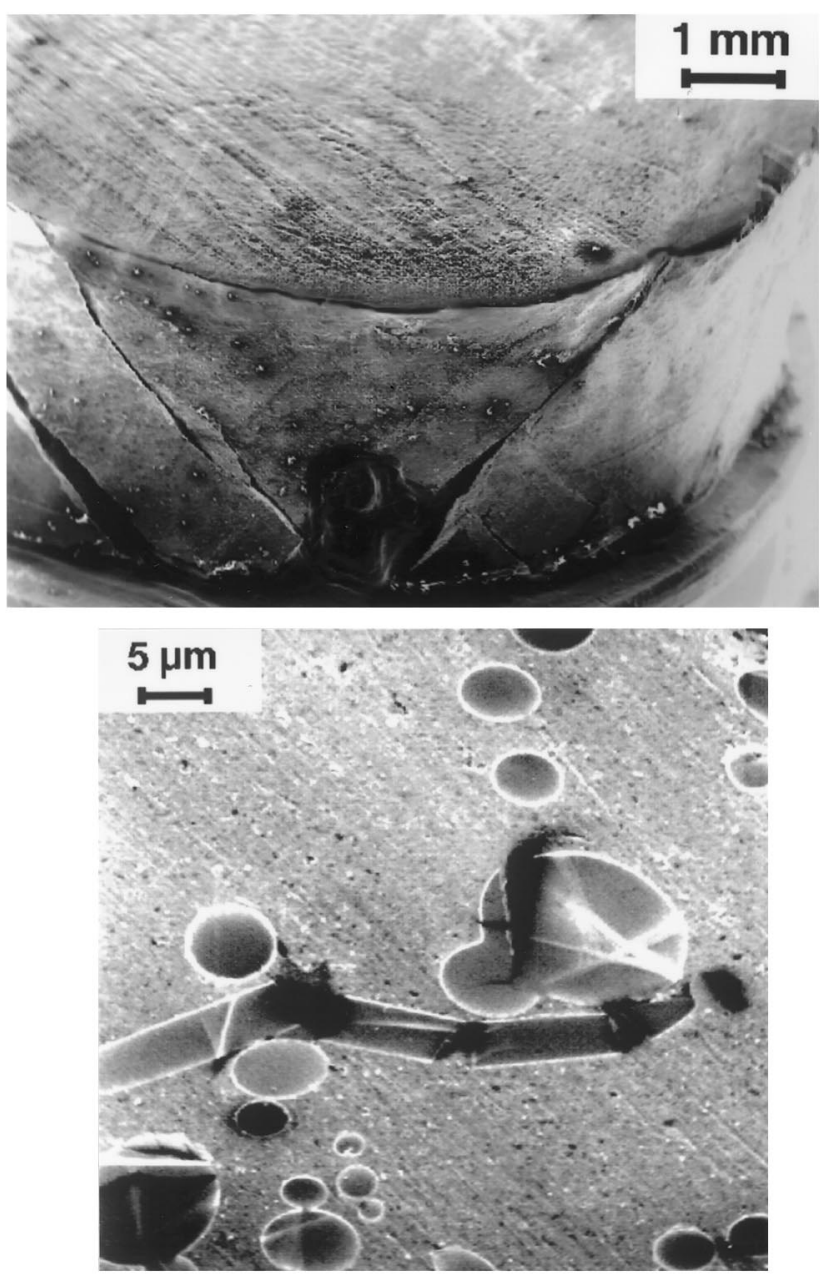

Fig. 9. (a) Cylindrical SF composite tested in the $\mathrm{P}$ direction to $\approx 45 \%$ strain showing characteristic failure mode. Compression axis vertical. (b) Cracking caused by fiber/fiber interaction.

from polished surfaces showed that the major damage mechanisms involved in compression testing were fiber fracture at small strains, followed by matrix microcracking at relatively large strains.

Three different types of fiber fracture events were observed, namely, shearing, buckling and fracture due to fiber/fiber interaction. The first two fiber fracture types occurred in fibers aligned parallel to the loading axis and are discussed in detail elsewhere [16]. A typical micrograph of fiber/fiber interaction fracture is shown in Fig. 9(b). This type of fracture was observed in fibers or fiber clusters adjacent to other fibers and resulted from bending of one fiber around the other(s).

Microcracks were often formed in regions where fibers clustered. The crack lengths were short and fibers effectively stopped these cracks before they became predominant fracture sites in the matrix. However, long cracks were occasionally observed to start near the edges of the samples and propagate through the bulk. 
Although the effect of strain rate on extent of fiber fracture and matrix microcracking could not be identified from polished sample cross-sections due to the random orientation of fibers, complete leaching of quasi-statically and dynamically deformed samples with dilute $\mathrm{HCl}$ to liberate the fibers and subsequent fiber fragment size analysis on the same $15 V_{\mathrm{f}}^{\mathrm{o}} \%$ composite material showed that higher strain rates produced smaller average fragment size compared with the quasistatic rates [16].

\subsubsection{Whisker-reinforced $M M C$}

Fracture occurred at $\approx 45^{\circ}$ to the loading direction in MMCs tested quasi-statically or dynamically and fractured samples closely resembled the SF sample shown in Fig. 9(a). Fracture at high strain rates in unreinforced alloy was very similar to the fracture of MMC. Microscopic studies on MMC and unreinforced alloy were carried out on fracture surfaces and on polished sections normal to the fracture surfaces. Unfractured unreinforced alloy samples tested near to the fracture strain were also observed by SEM and optical microscopy to clarify formation of the adiabatic shear bands commonly observed at high strain rate.

Fig. 10 shows a cylindrical unreinforced alloy sample in which a typical shear band formed from the edge of, and propagated into the bulk of, the sample at $\approx 45^{\circ}$ to the loading axis. In the shear band region the grains were highly distorted, essentially elongated through the band. Final failure occurred within the shear band via conventional ductile fracture involving growth and coalescence of fracture sites formed in the adiabatic shear band [17].

SEM micrographs of MMC fracture surfaces were clearly separated into two distinct types. These were: (a) regions smeared due to rubbing of the mating fracture surfaces during shear failure and (b) regions of ductile fracture which was preceded by debonding of whiskers.

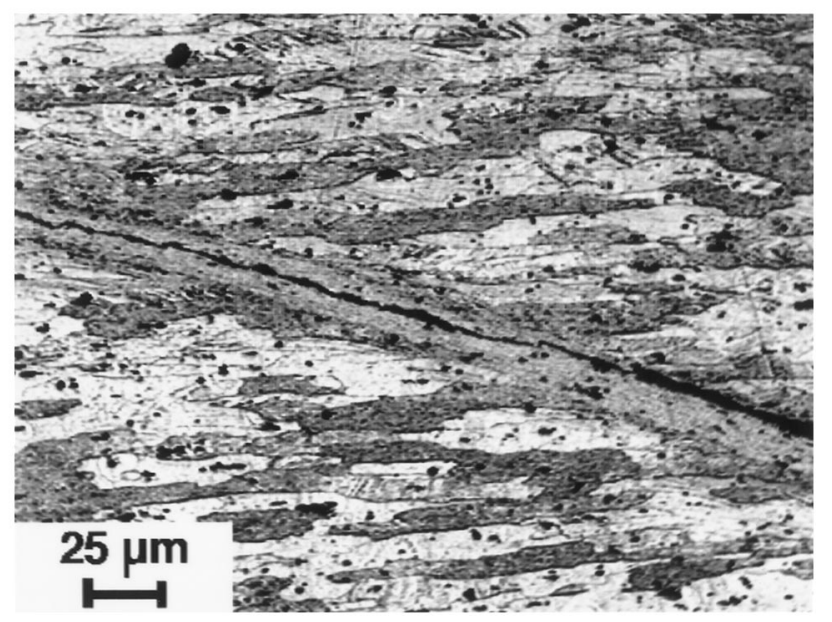

Fig. 10. Unreinforced 2024-T6 alloy showing shear band formation.
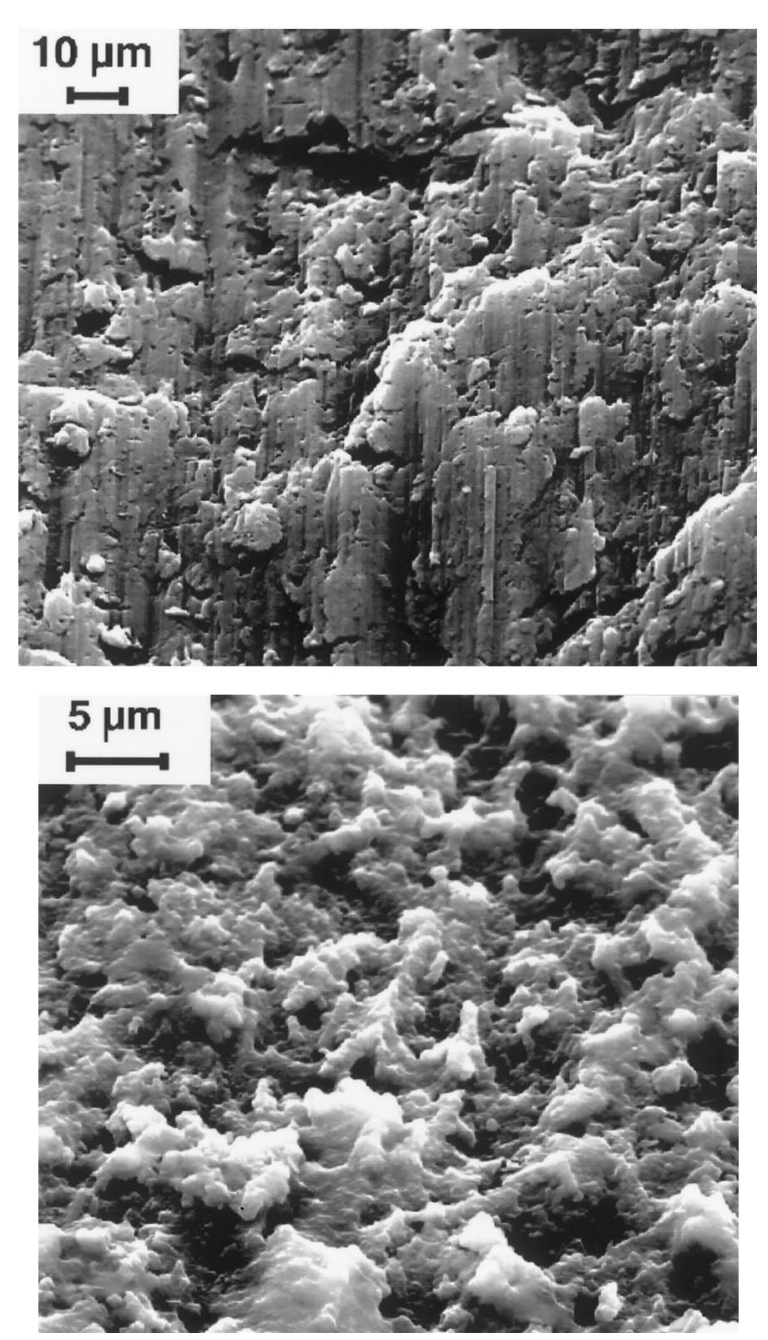

Fig. 11. (a) Characteristic fracture surface showing rubbing of mating surfaces during shear deformation. (Quasi-static test.) (b) Characteristic fracture surface showing ductile tearing during shear band formation and fracture. (Strain rate $2300 \mathrm{~s}^{-1}$ ).

These are illustrated in Fig. 11(a) and (b), for shear and ductile regions respectively. On fracture surfaces of quasi-statically tested MMC samples no clear ductile fracture region was detected.

In order to see more clearly the whisker orientation near to the shear band where fracture occurred, broken MMC samples were sectioned normal to the fracture surface and prepared metallographically for SEM examination. Fig. 12 shows a micrograph typical of dynamically tested samples. In a narrow zone near the fracture surface and in addition to severe fragmentation, fibers were observed to have suffered major rotations to lie parallel to the fracture surface. Previous studies on similar material showed that fracture occurred within shear bands [18], where the high local strains promote fiber debonding resulting, finally, in fracture of the sample. The observed debonded whiskers on the fracture surface clearly support this rationale. 


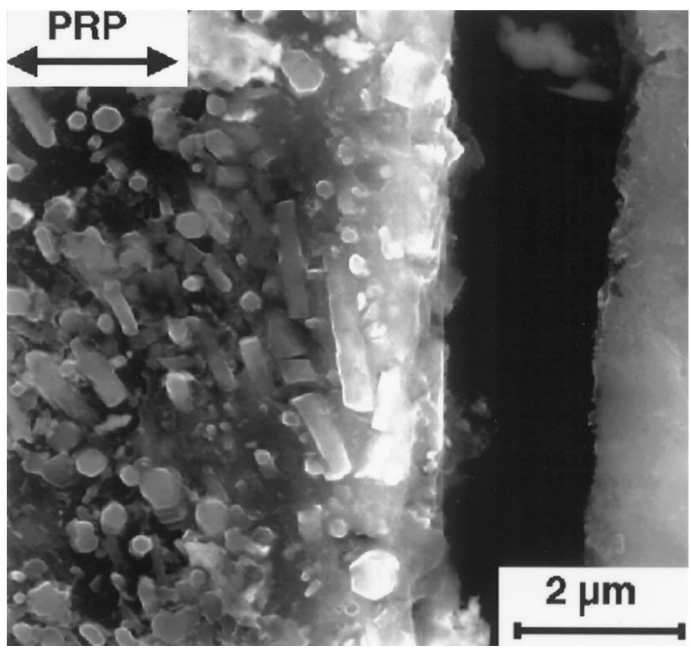

Fig. 12. SEM micrograph of opened shear band showing whisker fragmentation and gross realignment for primary reinforcement plane.

\subsubsection{Particulate-reinforced $M M C$}

Microscopic observations on polished samples showed that no fracture of the reinforcement or matrix occurred at quasi-static or dynamic strain rates until strains of $\approx 25 \%$, suggesting that, in the early stages of deformation, no appreciable damage occurred in these samples beyond what normally occurs in unreinforced material. This is also in accord with the observed stress/strain curves in which strain hardening is continuous at relatively large strain rates and strains. Fig. 13 shows a $\mathrm{SiC}_{\mathrm{p}}$ specimen strained to $25 \%$ at $1800 \mathrm{~s}^{-1}$ in which no damage can be seen, the structure resembles that of the as-received material.

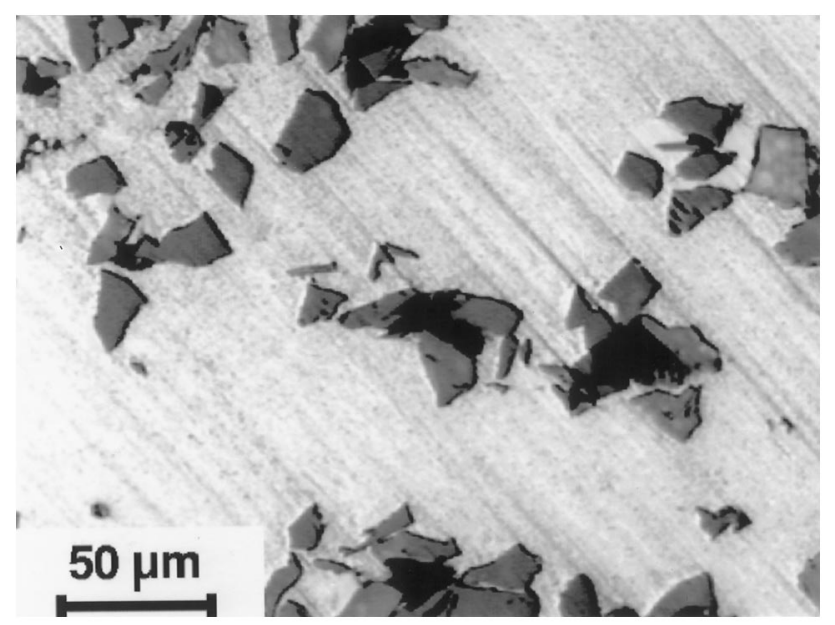

Fig. 13. $\mathrm{SiC}_{\mathrm{p}}$ composite strained to $25 \%$ at $1800 \mathrm{~s}^{-1}$ showing no apparent damage or particle cracking.

\section{Discussion}

\subsection{Rate sensitivity of unreinforced alloys and MMCs}

Common metallic materials such as $\mathrm{Al}$ [19] and $\mathrm{Cu}$ [20] have shown increased rate sensitive behavior at strain rates greater than $\approx 10^{3}-10^{4} \mathrm{~s}^{-1}$, a behavior which is generally interpreted as a change of deformation mechanism from thermally activated to drag controlled. Below this critical strain rate range, little or no rate sensitivity is observed. In the thermal activation controlled region, the flow stress $(\sigma)$ at a constant strain $(\varepsilon)$ can be written as [21];

$\sigma(\varepsilon)=\sigma_{0}(\varepsilon)+\sigma_{1}(\varepsilon) \log (\dot{\varepsilon})$

where $\sigma_{0}(\varepsilon)$ is the stress at unit strain rate, $\sigma_{1}$ is the strain dependent coefficient. By normalizing Eq. (4) with $\sigma_{0}$, we obtain;

$\frac{\sigma-\sigma_{0}}{\sigma_{0}}=\frac{\sigma_{1}}{\sigma_{0}} \log (\dot{\varepsilon})$

where $\frac{\sigma_{1}}{\sigma_{0}}$ may be considered as a parameter describing the rate sensitivity of the material. Experimental values of the rate sensitivity parameter of $\mathrm{Al}$ (containing $0.1-$ $0.2 \% \mathrm{Cu}$ ) have been reported as 0.034 [22] and 0.036 [21] at 5 and $6 \%$ strains respectively. Using Eq. (4), the rate sensitivity parameter of the present $\mathrm{Al}-1.2 \% \mathrm{Cu}$ unreinforced alloy is calculated to be 0.012 (at $5 \%$ strain) within the strain rate range studied, a rate sensitivity parameter which is almost three times less than the values reported for pure Al. This discrepancy may be ascribed to the higher alloy content and strength of the unreinforced alloy studied since Holt found that alloying elements tend to reduce rate sensitivity by increasing the athermal component of the stress [23]. Holt also showed that heat treatment can significantly reduce the rate sensitivity of metals; for example 6061-O has almost six times higher rate sensitivity parameter than 6061-T6 at 6\% strain [23]. T6 treatment has also been shown to have a similar effect on 7075 alloy and to reduce significantly the rate sensitivity of other Al alloys [23]. The observation that the present 2024-T6 alloy showed essentially no rate sensitivity is, therefore, in agreement with previous findings. However, 2024-O alloy is rate sensitive, having a rate sensitivity parameter of $\approx 0.015[15]$ at $6 \%$ strain in the range $10^{-2}-10^{3} \mathrm{~s}^{-1}$.

The above analysis of strain rate sensitivity of metallic materials only considers relatively small strains where any thermal softening effect is negligible. Increasing strains increase the effect of thermal softening at high strain rates due to relatively shorter deformation times. This effect can be clearly seen in Fig. 5(b) for 2024-T6 alloy in which increasing strains greatly reduced the high strain rate flow stress values compared 
to quasi-static values. On the other hand, the Al$1.2 \% \mathrm{Cu}$ alloy showed dynamic flow stress values which were initially higher than quasi-static flow stress values at lower strains $(\approx 5-10 \%)$, but the difference between them nevertheless decreased as the strain increased to $20 \%$. The difference in behavior of these two alloys has two sources: (i) a relatively higher rate sensitivity of the flow stress of $\mathrm{Al}-1.2 \% \mathrm{Cu}$ and (ii) higher strength of the 2024-T6 alloy compared with $\mathrm{Al}-1.2 \% \mathrm{Cu}$ alloy. The consequence of these differences is that more heat is generated and retained in the 2024-T6 alloy sample leading to more pronounced thermal softening at high strain rates.

Although many theoretical explanations for the increased flow stresses of metals at high strain rates have been advanced, the increased rate sensitivity of MMCs over unreinforced alloys remains a complex function of several parameters. Among other factors these may include: (1) local variation of strain rate near the matrix/reinforcement interface; (2) variation of the load carrying capacity of ceramic reinforcement itself at increasing strain rates; (3) interaction of dislocations with reinforcement; and (4) rate of damage formation.

As mentioned above, the strain rate in MMCs can reach very high local values at strain discontinuities present near the reinforcement. If the matrix alloy is itself rate sensitive at these locally attained strain- rates, the matrix strength will increase significantly and lead to an observed increase in rate sensitivity of the composite relative to the unreinforced alloy at increasing strain rates.

Previous studies [24,25] on bulk alumina have shown that the fracture strength in compression increased from quasi-static strain rates to moderate strain rates $\left(\approx 1000 \mathrm{~s}^{-1}\right)$ and a higher rate sensitivity of fracture strength is observed in many ceramic materials. Therefore, fibers based on these materials may themselves contribute their strain rate sensitivity to that of the composite at high strain rates.

Next, dislocation/reinforcement interaction may also be significant at high strain rates and the factors (1), (2) and (3), act cumulatively to increase the rate sensitivity of the MMC compared with the unreinforced alloy. However, the last factor, damage formation, is more likely to induce a reduced rate sensitivity in MMCs when compared with unreinforced alloy. For example, an increase of matrix shear stress increases the extent of fiber fracture in a short fiber MMC and strain localization or shear banding induces greater fiber fracture or debonding in a small region and leads to early fracture. The overall scenario, therefore, includes factors which tend to increase the rate sensitivity and others which tend to decrease it, the observed effect being a balance between the two. Damage analysis is, therefore, very important in understanding the rate sensitive behavior of MMCs.

\subsection{Effect of strain rate on MMCs and damage analysis}

SF-reinforced MMCs have shown clear effects of strain rate on flow stress. First, increasing strain rate produced an increased work hardening rate in the initial portion of the test but a decreasing rate thereafter. These effects, therefore, divide the stress/strain curve of an MMC into two distinct regions. The increased work hardening rate in the initial portion of stress/strain curves can be explained with reference to the first three parameters mentioned in the previous section. The reduced work hardening rate in the second region, however, is mainly due to increased damage formation and accumulation processes at high strain rates. Therefore, the rate sensitivity of MMCs is found to increase at small strains, $\approx 5 \%$, relative to unreinforced alloys but to decrease as the strain increases (see Fig. 2(a) and (b)). Typically observed damage consists of fiber fracture and matrix fracture (at larger strains).

In W-reinforced MMC, no strain rate-sensitivity was observed in the $5 \%$ flow stress data, similar to the unreinforced alloy. But, increasing the strain to $\approx 15 \%$, reduced the composite flow stress at increasing strain rates. Fracture of the MMC at relatively small strains (compared with unreinforced alloy ductility) demonstrates that thermal softening and subsequent damage accumulation occurred earlier in the MMC. This is due to the higher strength of the MMC and also to differences in thermal properties between the MMC and unreinforced alloy. It is interesting to note that previous work by Harding and Taya [2] on similar material showed totally different behavior and they reported an increase in strength with strain rate. However, it should be noted that their tests were in tension where shear banding is not found and where the strains to failure are very small (typically $4-5 \%$ ) so that high strain behavior can not be discerned. Typical damage formation observed in this MMC was shear localization leading to higher temperatures in the shear region and subsequently higher stress softening effects. Further, increased shear strain in the region also leads to increased fiber fracture and debonding at high strain rates.

P-reinforced MMCs showed rate sensitive flow stress behavior at all strain values studied. Microscopic observations showed that the reinforcement suffered no appreciable damage at increasing strains or strain rates, leading to no additional deformation modes compared with the unreinforced matrix and this is confirmed by the observed continuity in strain rate sensitivity found at increasing strain values. The measured rate sensitivity is a product of a combination of matrix rate sensitivity, local strain rate variation and a very minor effect due to interactions of dislocations with particles. 


\subsection{Re-loading tests}

These tests permit the detection and investigation of relaxation and heating effects on tested samples. Reloading tests on SF-MMC (Fig. 4) and its unreinforced alloy clearly demonstrated that both are sensitive to strain rate increments at the strain rates studied, $\approx$ $1000 \mathrm{~s}^{-1}$. However, the actual increase in stress in the higher strain rate tests might be reduced due to the strain rate history effect which is commonly observed in f.c.c. materials [26].

Whisker-reinforced MMCs showed a sudden increase in stress value followed by a reduction in stress during re-loading tests at the same strain rate, Fig. 7(a), an effect which is not believed to be due simply to dispersion effects in the bar. Except for the initial rise of stress, similar behavior was observed in the re-loading tests of unreinforced alloy. In both the MMC and unreinforced alloy, re-loading significantly increased the fracture strain proving that damage formation due to thermal softening and subsequent shear band formation had been delayed.

\section{Summary}

Three different MMCs were compression tested at quasi-static and moderate strain rates $\left(\approx 10^{3} \mathrm{~s}^{-1}\right)$ and the following significant differences in behavior were observed.

(1) Short fiber-reinforced MMCs showed significantly greater strain rate sensitivity of the flow stress than the unreinforced alloy, in both $\mathrm{P}$ and $\mathrm{N}$ directions. The measured rate sensitivity was found to decrease at increasing strain values compared with the unreinforced alloy.

(2) Whisker-reinforced MMC showed rate insensitive flow stress behavior at 5\% strain and experienced decreasing flow stress at high strain rates compared with quasi-static flow stress at increasing strains similar to unreinforced alloy. In this composite increasing strain rate slightly reduced the fracture strain.

(3) Particulate-reinforced MMC was found to be rate sensitive at all strain values studied.

(4) The increased rate sensitivity of short fiber- and particulate-reinforced MMCs and rate insensitivity of whisker-reinforced MMC, as compared with the corresponding unreinforced alloys, are related to the relative rate sensivity of the unreinforced alloys.

(5) The reduced rate sensitivity of some MMCs at increasing strain rates and strains is attributed to the damage formation processes. Microscopic damage analyses were conducted on MMC samples and the major damage processes were found to be fiber fracture followed by matrix cracking in short fiber reinforced
MMC and shear banding and complete fracture in whisker-reinforced MMC. No specific reinforcement related microscopic damage was detected in particulatereinforced MMCs.

(6) Re-loading tests were conducted and clearly revealed heating effects during testing.

\section{Acknowledgements}

The authors gratefully acknowledge financial support from the Army Research Office, award \# DAAH0495-2-0001. One of the authors (M. Guden) also gratefully acknowledges financial support from the Izmir Institute of Technology, Turkey.

\section{References}

[1] H. Kolsky, Proc. Phys. Soc. B62 (1949) 676.

[2] J. Harding, M. Taya, in: Proc. ICCM-6/ECCM-2, Elsevier, Amsterdam, 1987, p. 2.224.

[3] K. Cho, S. Lee, Y.W. Chang, J. Duffy, Metall. Trans. 22A (1991) 367.

[4] R. Vaziri, D. Delfosse, G. Pageau, A. Poursartip, Int. J. Impact Eng. 13 (1993) 329.

[5] C.C. Perng, J.R. Hwang, J.L. Doong, Mater. Sci. Eng. A171 (1993) 213.

[6] D.R. Chichili, K.T. Ramesh, Int. J. Sol. Struct. 32 (1995) 2609.

[7] S. Yadav, D.R. Chichili, K.T. Ramesh, Acta Metall. Mater. 43 (1995) 4453.

[8] G. Bao, Z. Lin, Acta Metall. Mater. 44 (1996) 1011.

[9] P.S. Follansbee, Metals Handbook 8, ASM, Metals Park, OH, 1985, pp. 198-203.

[10] R.M. Davies, Philos. Trans. 240A (1948) 375.

[11] E.D.H. Davies, S.C. Hunter, J. Mech. Phys. Sol. 11 (1963) 155.

[12] G. Ravichandran, G. Subhash, J. Am. Ceram. Soc. 77 (1994) 263.

[13] D.J. Parry, P.R. Dixon, S. Hodson, N. Al-Maliky, J. Phys. IV 4 (1994) C8-107.

[14] 'Aluminum Standards and Data', Aluminum Association, Washington DC, 1984.

[15] U.S. Lindholm, L.M. Yeakley, J. Mech. Phys. Sol. 13 (1965) 41.

[16] M. Guden, I.W. Hall, Mater. Sci. Eng. A232 (1997) 1.

[17] M.A. Meyers, J. Phys. III 4 (1994) C8-597.

[18] K. Cho, S. Lee, W.B. Choi, I. Park, Advanced Composites '93 Int. Conf. on Advanced Composite Materials, The Minerals, Metals and Material Society, 1993.

[19] R. Dowling, J. Harding, J.D. Campbell, J. Inst. Met. 98 (1970) 215.

[20] P.S. Follansbee, in: L.E. Murr, K.P. Staudhammer and M.A. Meyers (Eds.), Metallurgical Applications of Shock Wave and High-Strain Rate Phenomena, Dekker, New York, 1986, p. 451.

[21] U.S. Lindholm, J. Mech. Phys. Sol. 12 (1964) 317.

[22] J.L. Chiddister, L.E. Malvern, Exp. Mech. (1963) 81.

[23] D.L. Holt, S.G. Babcock, S.J. Greenm, C.J. Maiden, Trans. ASM 60 (1967) 152.

[24] J. Lankford, J. Mater. Sci. 12 (1967) 791.

[25] J. Lankford, J. Am. Ceram. Soc. 64 (1981) C33.

[26] P.E. Sensey, J. Duffy, R.H. Hawley, J. Appl. Mech. 45 (1978) 60. 